Research Paper

\title{
Knockdown of hTERT and Treatment with BIBR1532 Inhibit Cell Proliferation and Invasion in Endometrial Cancer Cells
}

\author{
Weimin Kong1, Nenan Lv11, Weiya Z. Wysham², Dario R. Roque², Tongqing Zhang1, Simeng Jiao1, Dan \\ Song, Jiao Chen ${ }^{1}$, Victoria L. Bae-Jump ${ }^{2,3 凶}$, Chunxiao Zhou ${ }^{2,3 凶}$ \\ 1. Department of Gynecological Oncology, Beijing Obstetrics and Gynecology Hospital affiliated to Capital Medical University. Beijing, P. R. China. \\ 2. Division of Gynecological Oncology, Department of Obstetrics and Gynecology, University of North Carolina at Chapel Hill, Chapel Hill, NC, \\ United States of America. \\ 3. Lineberger Comprehensive Cancer Center, University of North Carolina at Chapel Hill, Chapel Hill, NC, United States of America. \\ $\triangle$ Corresponding authors: Chunxiao Zhou MD PhD. Assistant Professor. Division of Gynecologic Oncology, Department of Obstetrics and Gyne- \\ cology, University of North Carolina at Chapel Hill, Chapel Hill, NC, United States of America. Phone 919-966-3270 Email: czhou@med.unc.edu.
}

() 2015 Ivyspring International Publisher. Reproduction is permitted for personal, noncommercial use, provided that the article is in whole, unmodified, and properly cited. See http://ivyspring.com/terms for terms and conditions.

Received: 2015.06.26; Accepted: 2015.08.28; Published: 2015.11.01

\begin{abstract}
Telomerase activity and expression of the catalytic protein hTERT are associated with cell proliferation and advanced stage in endometrial cancer. Our objective was to evaluate the effect of inhibition of $\mathrm{hTERT}$ by siRNA and BIBR 1532 on cell growth, apoptosis and invasion in endometrial cancer cells. Knockdown of hTERT or treatment of the cells with BIBR 1532 decreased telomerase activity, inhibited cell proliferation, induced apoptosis, and reduced cell invasion in Ishikawa and ECC-1 cells. Either hTERT siRNA or BIBR 1532 in combination with paclitaxel promoted a synergistic inhibitory effect on cell growth through induction of Annexin $V$ expression and a remarkable reduction in cell invasion through reduction of protein expression of MMP9, MMP2, and MMP3. Increased telomerase activity and hTERT protein expression by transfections enhanced the protein expression of MMPs and increased the cell invasion ability. BIBR1532 significantly antagonized cell invasion induced by increased hTERT expression. These findings suggest that telomerase and hTERT facilitate cell invasion via MMP family in human endometrial cancer cells.
\end{abstract}

Key words: telomerase, hTERT, endometrial cancer, apoptosis, paclitaxel.

\section{Introduction}

Telomerase is a ribonucleoprotein reverse transcriptase that adds DNA sequence repeats to the $3^{\prime}$ end of DNA strands in the telomere regions, which is composed of two main subunits: the catalytic protein hTERT and an RNA template hTR [1]. The activity of telomerase and expression of hTERT are observed in $85-90 \%$ of most cancers and contribute to the increased cell proliferation, cellular immortality, carcinogenesis and cancer progression, while activity of telomerase and hTERT expression are usually repressed in most normal tissues[2,3]. This difference in telomerase activity and hTERT expression in normal and cancer cells makes telomerase an ideal approach for reducing cancer growth [4, 5]. Furthermore, hTERT may serve as an attractive target in inhibition of telomerase as the reduction of hTERT has been shown to inhibit telomerase activity and block cancer cell growth effectively using RNA interference and pharmacological agents [5, 6]. In fact, several telomerase inhibitors such as imetelstat (GRN163L) have demonstrated a favorable pharmacokinetic profile with minimal side effects in phase 1 and 2 clinical trials $[7,8]$.

Endometrial cancer is the most common gynecologic malignancy in women in the Western world, and it is the fourth most common cancer among 
women in the United States [9]. It has been confirmed that more than $85 \%$ of human endometrial carcinomas express telomerase activity, and the level of telomerase activity has been correlated with advanced stage disease and with pelvic lymph node metastasis [9]. Higher telomerase activity has also been shown to represent a deregulation of the cell cycle related to $S$ phase and a higher degree of malignancy in endometrial cancer [10]. The level of hTERT mRNA expression and telomerase activity showed a linear association. Furthermore, expression of hTERT was closely associated with proliferation, apoptosis, high grade disease and worse prognosis in endometrial cancer [11-13]. The activity of radiation-induced telomerase might be associated with radiosensitivity in endometrial cancer cells [14]. Antisense hTERT has been previously found to decrease the activity of telomerase, inhibit cell proliferation and reduce tumor growth in vitro and in vivo in endometrial cancer [15].

BIBR1532 (BIBR) is a noncompetitive, non-nucleoside pharmacological small molecule inhibitor that inhibits telomerase activity by binding to the active site of hTERT, with a strong ability to inhibit cell viability effectively across several cancer cell types including breast, lung, leukemia, ovarian, germ cell tumors and chondrosarcoma [1, 16-18]. Recent studies have demonstrated synergistic effects in vitro with combinations of arsenic and chemotherapeutic agents, such as paclitaxel and cisplatin [1, 8, 16, 19]. We have recently shown that inhibition of cell proliferation by PTEN, arsenic, metformin and rapamycin is associated with a decrease in telomerase activity and expression of hTERT mRNA, suggesting that telomerase and hTERT are cell proliferative markers in endometrial cancer cells [20-24]. In the present study, we investigated the effect of knockdown of hTERT by siRNA and treatment with BIBR1532 in combination with paclitaxel on cell proliferation, apoptosis and invasion in endometrial cancer cell lines. The results indicate that downregulation of hTERT expression results in reduction of cell proliferation and invasion and promotes the anti-cancer effects of paclitaxel on endometrial cancer cells.

\section{Material and Methods}

\section{Cell culture and reagents}

The endometrial cancer cell lines, ECC- 1 and Ishikawa, were used. The ECC-1 cell line was maintained in RPMI 1640 supplemented with 5\% FBS, $300 \mathrm{mM}$ L-glutamine, $5 \mu \mathrm{g} / \mathrm{ml}$ bovine insulin, 10,000 $\mathrm{U} / \mathrm{ml}$ penicillin and $10,000 \mu \mathrm{g} / \mathrm{ml}$ streptomycin. Ishikawa cells were grown in MEM supplemented 5\% FBS, $300 \mathrm{mM}$ L-glutamine, 10,000 U/ml penicillin and $10,000 \mu \mathrm{g} / \mathrm{ml}$ streptomycin. Both the cell lines were cultured at $37^{\circ} \mathrm{C}$ in a humidified atmosphere with $5 \%$ CO2. MTT (3-(,5-dimethylthiazol-2-yl)-2,5-diphenyltetrazolium bromide) and RNase A were purchased from Sigma (St. Louis, MO). The anti-MMPs and anti-a-tubulin antibodies were purchased from Cell Signaling (Beverly, MA). The anti-hTERT antibody and Annexin V FITC kit were purchased from Abcam (Cambridge, MA). The Enhanced chemiluminescence Western blotting detection reagents were purchased from GE Health care (GE Healthcare Life Sciences, Piscataway, NJ). BIBR1532 was purchased from Cayman (Ann Arbor, MI) and dissolved in DMSO. All other chemicals were purchased from Sigma (St Louis, $\mathrm{MO})$.

\section{MTT proliferation assay}

The ECC-1 and Ishikawa cells were plated and grown in 96-well plates at a concentration of 4000 cells/well for 24 hours. These cells were subsequently treated with BIBR or hTERT siRNA for 48 hours. Viable cell densities were determined by metabolic conversion of the dye MTT. MTT $(5 \mathrm{mg} / \mathrm{ml})$ was added to the 96-well plates at $5 \mu \mathrm{l} /$ well, followed by an additional hour of incubation. The MTT reaction was terminated through the addition of 100 $\mathrm{pl}$ of DMSO. The results were read by measuring absorption at $570 \mathrm{~nm}$. The effect of BIBR or hTERT siRNA was calculated as a percentage of control cell growth obtained from DMSO treated cells grown in the same 96-well plates. Each experiment was performed in triplicate and repeated three times to assess for consistency of results.

\section{Invasion assay}

Cell invasion assays were performed using 96-well HTS transwells (Corning Life Sciences ,Tewksbury ,MA) coated with 0.5-1X BME (Trevigen Inc., Gaithersburg, MD). Endometrial cancer cells or the cells transfected with siRNA hTERT (50,000/well) were starved for 12 hours and then seeded in the upper chambers of the wells in $50 \mu \mathrm{l} \mathrm{FBS-free} \mathrm{medium.}$ The lower chambers were filled with $150 \mu$ regular medium or with BIBR. The plate was incubated for 24 hours at $37^{\circ} \mathrm{C}$ to allow cell invasion into the lower chamber. After washing the upper and lower chambers with PBS once, 100 ul Calcein AM solution was added into lower chamber and incubated at $37^{\circ} \mathrm{C}$ for 30-60 minutes. The lower chamber plate was measured using a plate reader for reading fluorescence at EX/EM 485/520 nm. The experiments were repeated three times

\section{Annexin $\mathbf{V}$ assay}

Annexin V/PI double staining was performed to evaluate the induction of apoptosis. ECC-1 and Ishikawa cells $\left(2 \times 10^{5} /\right.$ well $)$ were seeded in 6 -well plates. The cells were treated with various concentrations of 
paclitaxel or transfected with siRNA hTERT or combination for 24 hours. Then cells were harvested using trypsin, washed with PBS, and suspended in $100 \mu \mathrm{L}$ binding buffer. 1ul of annexin V-FITC $(100 \mathrm{ug} / \mathrm{ml})$ and $0.5 \mathrm{uL}$ of propidium iodide $(2 \mathrm{mg} / \mathrm{ml})$ were added in the binding buffer in the dark for 15 minutes. Cell apoptosis was detected by Cellometer (Nexelom, Lawrence, MA) and subsequently analyzed by FCS4 express software. The experiments were repeated three times

\section{Telomerase activity assay}

Telomerase activity in culture cells was assessed with a PCR-based telomeric repeats amplification protocol (TRAP) as previously reported [25, 26]. Briefly, $1 \times 105$ cell pellets were stored at $-80^{\circ} \mathrm{C}$ until lysis was performed. Samples were homogenized with 1× CHAPS lysis buffer (3-[(3-cholamidopropyl)dimethyl-ammonio]-1-propa ne-sulfonate) at $1 \times 105$ cells/100 $\mu$ l, incubated on ice for $30 \mathrm{~min}$ and centrifuged at $13,000 \mathrm{~g}$ for $21 \mathrm{~min}$ at $4^{\circ} \mathrm{C}$. The supernatant was transferred into a fresh tube, and the protein concentration was measured by ABC kit (Bio-Rad, Hercules, CA). Between 0.25 and $0.5 \mu \mathrm{g}$ of protein from the cell extract was mixed with 32P-labeled TS primer in a $50 \mu \mathrm{l}$ reaction mixture. After $30 \mathrm{~min}$ of incubation at $30^{\circ} \mathrm{C}, \mathrm{PCR}$ amplification was then performed with 27 cycles at $94^{\circ} \mathrm{C}$ for $30 \mathrm{~s}$ and $59^{\circ} \mathrm{C}$ for $30 \mathrm{~s}$. The PCR products were separated by electrophoresis on $10 \%$ polyacrylamide non-denaturing gels. Phosphor-Imager and Imagequant software from Molecular Dynamics (Sunnyvale, CA) were used to quantify the band intensities. The experiments were repeated three times.

\section{Real-time RT-PCR for hTERT}

Total RNA was isolated using the QiAGEN kit (Valencia, CA). The reverse transcription and PCR reactions were performed using the TaqMan Gold one-step RT-PCR kit in the ABI Prism 7700 Sequence Detection System (Applied Biosystems, Foster City, CA). Reverse transcription was carried out at $48^{\circ} \mathrm{C}$ for $30 \mathrm{~min}$. The PCR condition consisted of a 10-min step at $95^{\circ} \mathrm{C}$ and 40 cycles at $95^{\circ} \mathrm{C}$ for $15 \mathrm{~s}$ and $65^{\circ} \mathrm{C}$ for 1 min. A housekeeping control gene acidic ribosomal phosphoprotein P0 (RPLP0, also known as 36B4) was used as an internal control to correct for differences in the amount of RNA in each sample. Primers and fluorogenic probes for hTERT and RPLP0 have been described previously [27]. The standard curve for hTERT was generated by using dilutions of a known amount of cRNA synthesized by in vitro transcription of a cloned fragment. The normalized level of hTERT in each sample was estimated by a ratio of the hTERT level to the RPLP0 level, as described previously[27]

\section{hTERT siRNA transfection}

Transfection of siRNA was performed using ABM hTERT siRNA (Applied Biological Materials Inc, Canada) and QIAGEN HiperFect Transfection Reagent. In brief, the cells were plated at a density of $2 \times$ $10^{5}$ cells/well in 6-well plates or $5 \times 10^{3}$ cells/well in 96-well plates. The transfection complexes containing $150 \mathrm{ng}$ of hTERT siRNA and 6 ul HiperFect Transfection Reagent for 12-well plate cells or $25 \mathrm{ng}$ of hTERT siRNA and $1 \mathrm{ul}$ of HiperFect Transfection Reagent for 96-well plate cells were added into the media. A scramble siRNA was used as the negative control. The impact of siRNA transfection on cell proliferation was evaluated by MTT assay. The experiments were repeated three times.

\section{hTERT retroviruses infection}

Lenti-hTERT and Lenti-empty vector were purchased from ABM. The viral infection was performed following ABM's instructions. Briefly, Ishikawa cells were exposed to the concentrated virions in the presence of the polybrene to infect cells on 6 well plates. After 6-8 hours, the virions mix was removed and fresh medium was added. Cells were selected continuously in puromycin $(2 \mathrm{ug} / \mathrm{ml})$.

\section{Western blotting analysis}

Cells were washed twice with PBS and then collected and lysed with RIPA buffer after treatment. The protein concentrations in the cell lysates were measured using a BCA Protein Assay Kit (Pierce, Rockford, IL). Protein samples were separated with $12 \%$ SDS-PAGE gel and transferred onto PVDF membranes (GE Healthcare Life Sciences, Piscataway, New Jersey). The membranes were blocked with $5 \%$ nonfat dry milk and probed with primary antibodies overnight at $4^{\circ} \mathrm{C}$. The membranes then were washed and incubated with a secondary peroxidase conjugated antibody for $1 \mathrm{~h}$. Antibody binding was detected using Amersham ECL detection reagents (GE Healthcare Life Sciences, Piscataway, New Jersey) via X films. All blots were stripped and reprobed with polyclonal anti- $\beta$-actin antibody or anti-a-tubulin antibody to ascertain equal loading of proteins. Each experiment was repeated three times to assess for consistency of results

\section{Statistics}

The Student's t-test was used for comparisons where indicated. Statistical significance was given at $p$ $<0.05$. Analysis of synergy was calculated using CalcuSyn software (Biosoft, Cambridge, UK). Combination index $(\mathrm{CI})<1$ indicates synergism, $\mathrm{CI}=1$ indicates additive effects and CI $>1$ indicates antagonism. 


\section{Results}

\section{Downregulation of telomerase activity by hTERT siRNA and BIBR 1532}

We first examined whether hTERT siRNA could effectively knock down hTERT protein expression in ECC-1 and Ishikawa cells. As shown in Fig 1A, transfection of the cells with hTERT siRNA and treatment of the cells with BIBR (50 uM) for 48 hours resulted in a marked decrease in hTERT protein expression by $65-70 \%$ in both cell lines. Real time PCR results showed both hTERT siRNA and BIBR resulted in $65-80 \%$ inhibition of hTERT mRNA expression in ECC-1 cells and 59-64\% inhibition in Ishikawa cells as compared with control cells (Fig 1B). Furthermore, our results from PCR-TRAP assays showed that knockdown of hTERT significantly reduced telomerase activity in both cell lines (Fig 1C). These results suggested that either hTERT siRNA or BIBR treatment effectively decreased telomerase activity in our endometrial cancer cells.

\section{Effect of hTERT siRNA and BIBR1532 on cell growth and apoptosis}

We previously showed inhibition of cell proliferation was associated with a decrease in telomerase activity in endometrial cancer [20-24]. Thus, we next assessed the effects of knockdown hTERT and BIBR treatment on cell viability and apoptosis in ECC-1 and Ishikawa cells. As we expected, hTERT siRNA or BIBR (50 uM) decreased cell proliferation in both cell lines after 48 hours of treatment. hTERT siRNA seems to have much more inhibitory effect than treatment with BIBR (Fig 2A). To further analyze the role of hTERT siRNA and BIBR on the apoptosis pathways, we used flow cytometry and western blotting to determine the expression of Annexin $\mathrm{V}$ and A
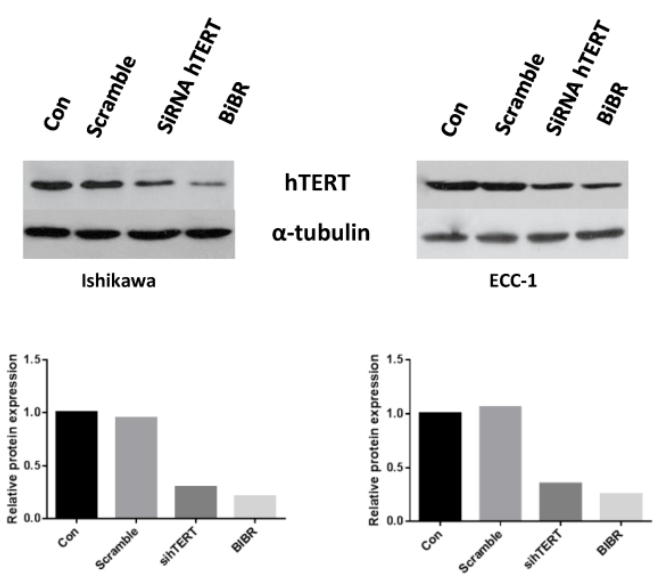

cleaved-caspase 3 in both cell lines, respectively. hTERT siRNA or BIBR (50 uM) significantly resulted in an increase in the expression of Annexin $\mathrm{V}$ and cleaved caspase 3 compared with control groups after 24 hours treatment (Fig 2B and C). hTERT siRNA seemed to induce more apoptosis than treatment with BIBR $50 \mathrm{uM}$ in both cell lines, although the difference did not reach statistical significance. These results suggested that inhibition of cell growth by siRNA or BIBR involved the mitochondrial apoptosis pathway in ECC-1 and Ishikawa cells.

\section{hTERT siRNA and BIBR 1532 enhanced the sensitivity to paclitaxel}

The inhibition of telomerase activity by telomelysin and BIBR has been shown to increase sensitivity to paclitaxel in head and neck squamous cell carcinoma and breast cancer $[8,28]$. To determine whether hTERT siRNA or BIBR increases sensitivity to paclitaxel in endometrial cancer cells, we treated the cells with paclitaxel for 48 hours after transfection of hTERT siRNA for 24 hours. Cell proliferation was assessed by MTT assay. Both cell lines were sensitive to paclitaxel treatment. The results showed that the combination of hTERT siRNA with different concentrations of paclitaxel exhibited synergistic inhibitory effects in ECC-1 and Ishikawa cells compared with paclitaxel alone (Fig 3A and 3B, CI $<1$ ). We next examined whether BIBR displayed the same synergy in paclitaxel treated cells. Both cell lines were treated with $50 \mathrm{uM}$ BIBR and various concentrations of paclitaxel for 48 hours. MTT results also demonstrated that the combination of BIBR with paclitaxel showed synergistic inhibitory effects (Fig 3C and 3D, $\mathrm{CI}<1)$.
C

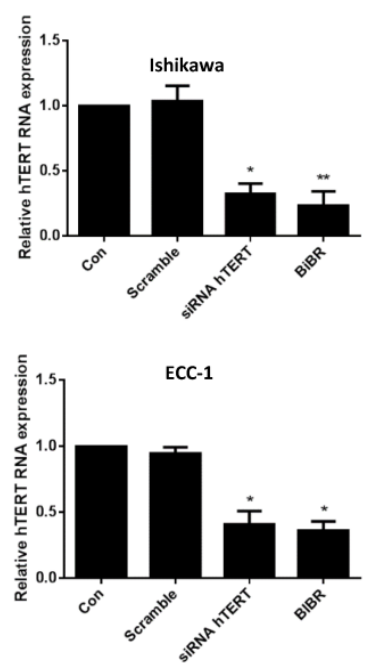

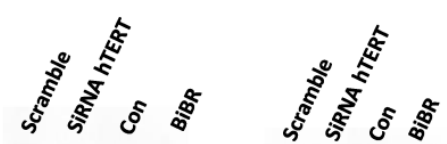

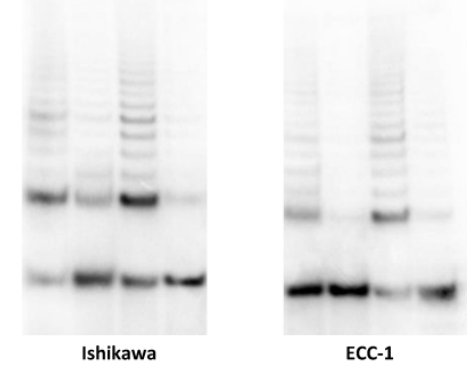

Figure 1. hTERT siRNA and BIBR1532 decrease telomerase activity and hTERT mRNA expression. Endometrial cancer cells lines, ECC-1 and Ishikawa, were transfected with hTERT siRNA and treated with BIBR 1532 for 48 hours. hTERT siRNA and BIBR 1532 reduced hTERT protein expression (A), hTERT mRNA expression (B) and telomerase activity $(C)$. Data shown are representative of 3 independent experiments $(* \mathrm{p}<0.05, * * \mathrm{P}<0.01)$. 
A

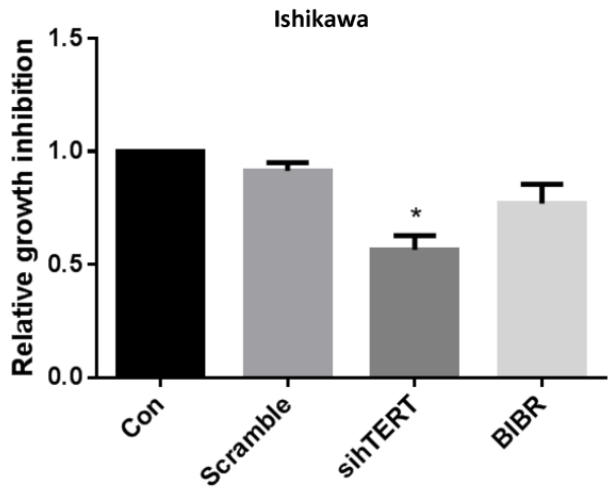

B

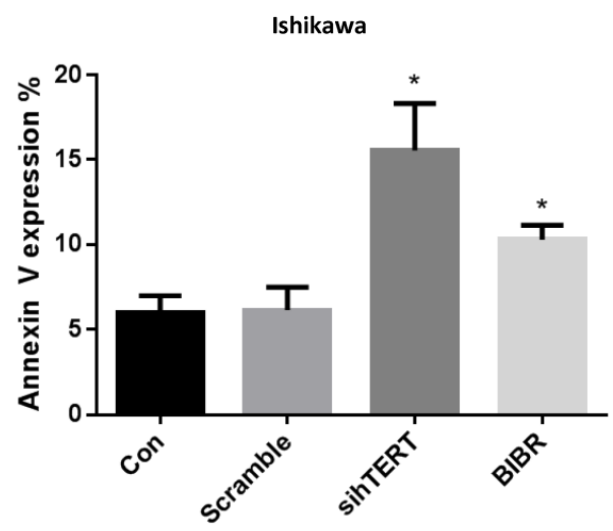

C

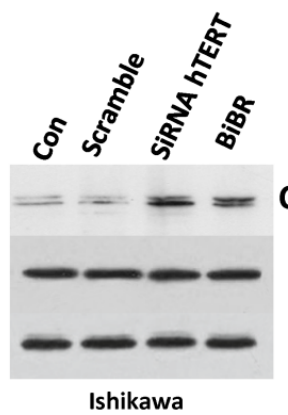

Caspase 3-Cleaved

Caspase 3

$\boldsymbol{\alpha}$-tubulin

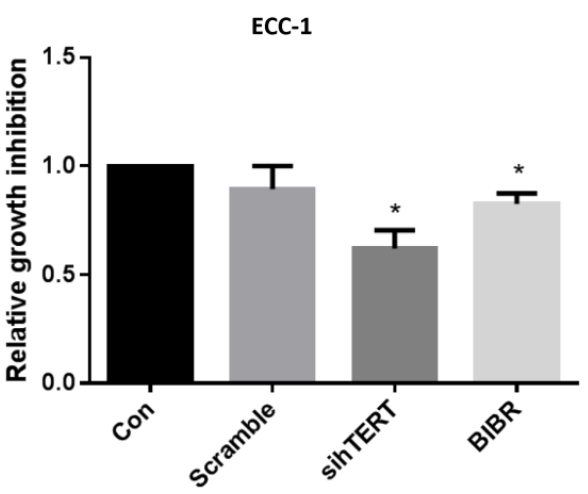

ECC-1

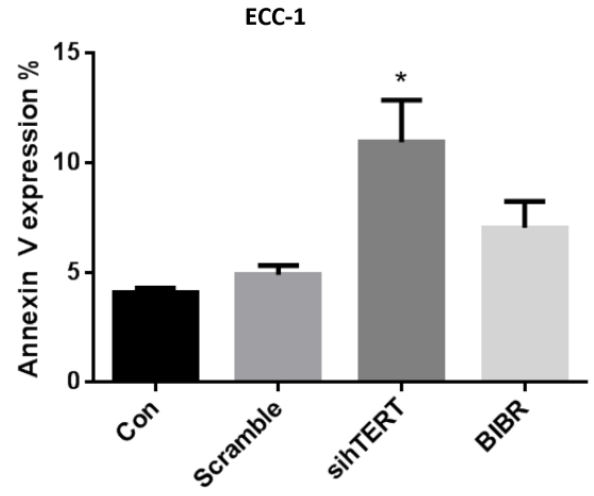

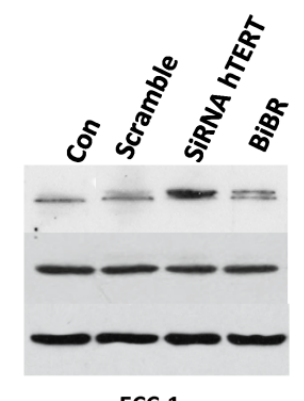

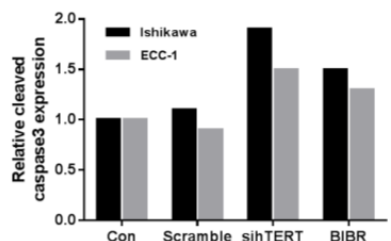

Figure 2. hTERT siRNA and BIBR1532 inhibit cells growth and induce apoptosis. ECC-1 and Ishikawa cells were transfected with hTERT siRNA and treated with BIBR 1532 for 48 hours. Cell proliferation was assessed by MTT assay. Inhibition of hTERT decreased cell proliferation (A). ECC-1 and Ishikawa cells were transfected with siRNA for hTERT or treated by BIBR1532 for 24 hours. The apoptosis were detected using Annexin $V$ assay (B) and Western blotting (C). hTERT siRNA or BIBR1532 induced expression of Annexin $V$ and cleaved caspase 3 in both cell lines. Data is shown as mean + SEM of triplicates $(* \mathrm{p}<0.05)$.

To further address the underlying mechanism of synergistic inhibition by hTERT siRNA or BIBR in combination with paclitaxel, we measured Annexin V expression and cleaved caspase 3 activity by flow cytometry and ELISA assay following treatment of ECC-1 and Ishikawa cells for 24 hours. Both cell lines behaved similarly towards the hTERT siRNA, BIBR or combination with paclitaxel treatments. Treating cells with paclitaxel $(5 \mathrm{nM})$ alone or in combination with hTERT siRNA or BIBR for 24 hours resulted in a significant increase in the expression of Annexin $\mathrm{V}$ in both cell lines. Paclitaxel in combination with hTERT siRNA was more effective than paclitaxel in combination with BIBR1532 in inducing Annexin V expression, with a three- to four-fold increase in Annexin $\mathrm{V}$ expression in both cells compared with respective control cells (Fig 3E and 3F). Similar results were also obtained using cleaved caspase 3 activity to measure the treatments in both cell lines (data not shown). These results suggested that combination treatments resulted in an increase in apoptotic cells.

\section{hTERT siRNA and BIBR1532 inhibited cell in- vasion}

The effect of hTERT siRNA and BIBR on the invasion of endometrial cancer cells was analyzed by a transwell invasion assay. After 24 hours of hTERT siRNA or BIBR (50uM) treatment, the migratory capacity of ECC-1 and Ishikawa cells was reduced by $23-33 \%$ in hTERT siRNA groups and $13-16 \%$ in BIBR groups compared with control groups (Fig 4A and 4B). Western blotting revealed that hTERT siRNA and 
BIBR decreased protein expression of matrix metallopeptidases (MMP2, MMP3 and MMP9), hallmarks of cell invasion (Fig 4C). To further address the role of hTERT knockdown in cell invasion, we hypothesized that the overexpression of hTERT protein could increase cell invasion and decreased MMP expression induced by hTERT siRNA and BIBR. A Lenti-hTERT expression vector was expressed in Ishikawa cells after puromycin selection. As shown in Figure 4D, a significant increase of hTERT protein expression and telomerase activity was observed in this transfected cell line. In support of our hypothesis, overexpression of hTERT significantly increased protein expression of
MMP2, MMP3 and MMP9 in transfected Ishikawa cells (Fig 4E). We also found that overexpression of hTERT enhanced the invasiveness, and treatment with BIBR eliminated approximately $25 \%$ of the invasion ability in the cells (Fig 4F), thus, suggesting that hTERT was involved in cell invasion in endometrial cancer cells. Furthermore, we examined the effects of paclitaxel in combination with hTERT siRNA and BIBR treatments on cell invasion. Treatment of cells with paclitaxel plus hTERT siRNA or BIBR significantly reduced cell invasion compared with single agent alone groups (hTERT siRNA, BIBR and paclitaxel) in both cell lines (Fig 4G and H).
A

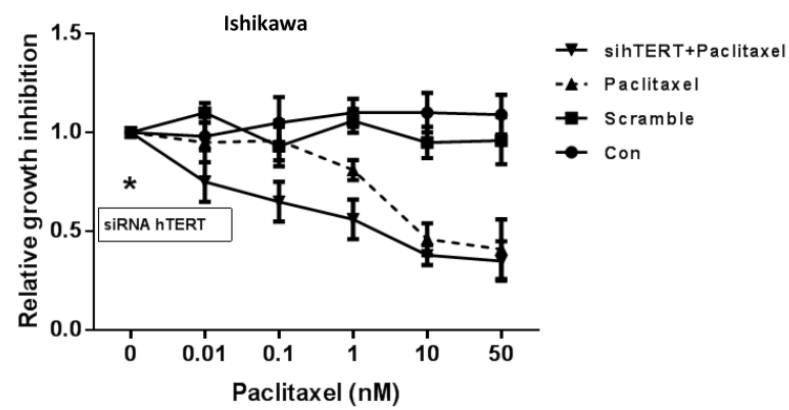

C

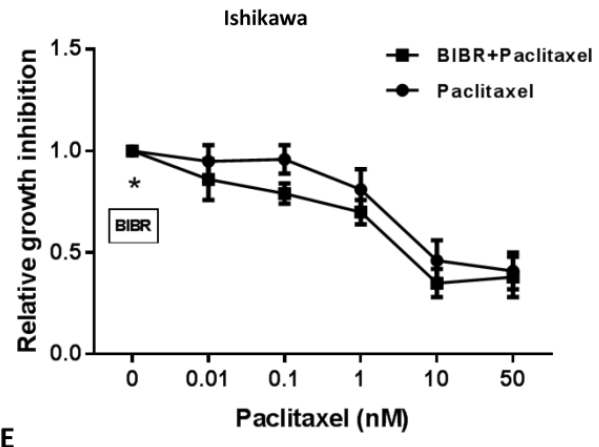

E

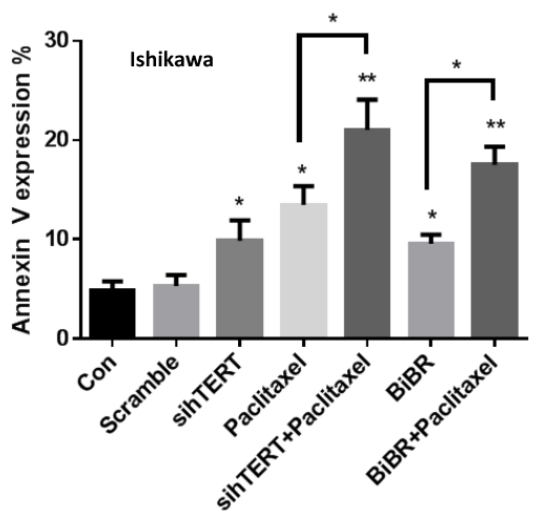

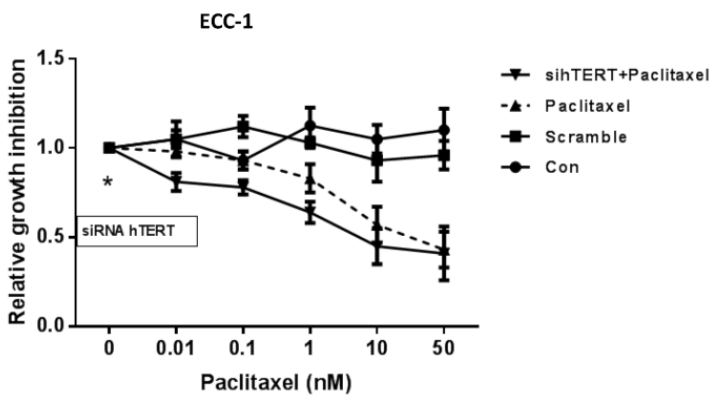

D

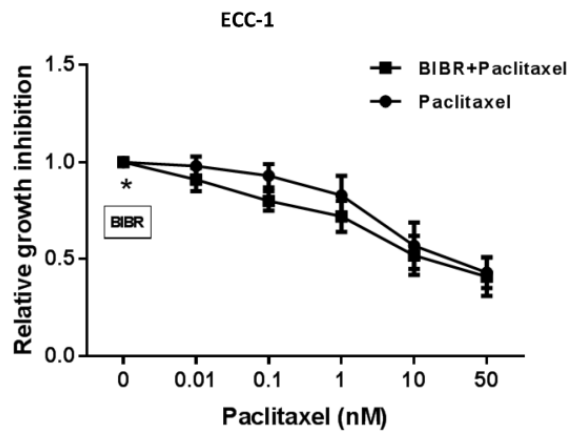

$\mathbf{F}$

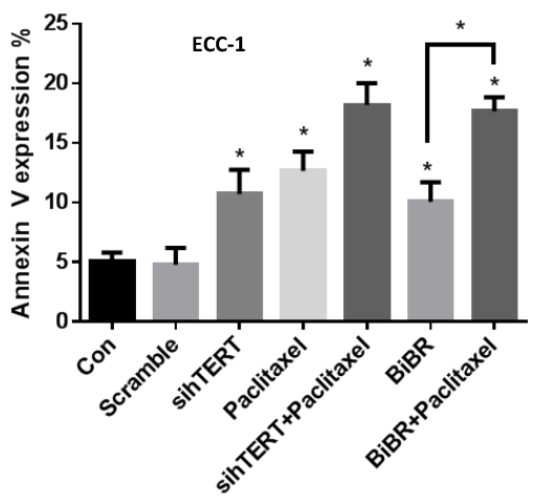

Figure 3. hTERT siRNA and BIBR 1532 increase the sensitivity to paclitaxel. The cells were treated with hTERT siRNA and BIBR 1532 for 48 hours. The cell proliferation was assessed by MTT assay. hTERT siRNA and BIBR 1532 synergistically increased cell sensitivity to paclitaxel in the both cells (A-D, Cl<1). ECC-1 and Ishikawa cells were treated with BIBR 1532 or transfected with hTERT siRNA for 24 hours. Annexin $V$ expression was assessed by flow cytometry (E and F). The combination therapy with paclitaxel effectively induced annexin $\mathrm{V}$ expression in both cells. Data are representative of triplicate determinants $\pm \mathrm{SE}$. $(* \mathrm{p}<0.05)$. 

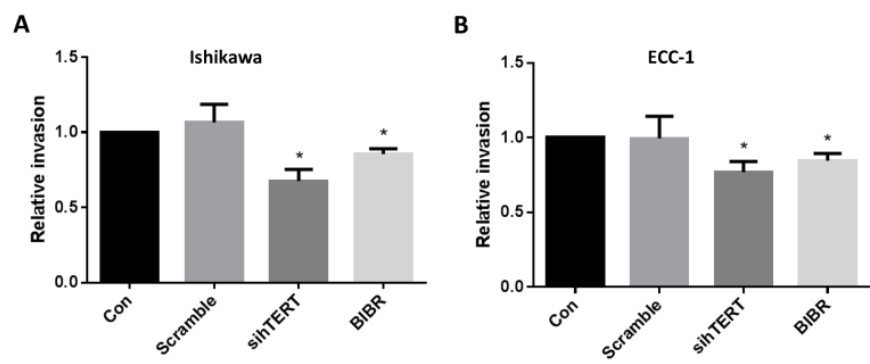

D

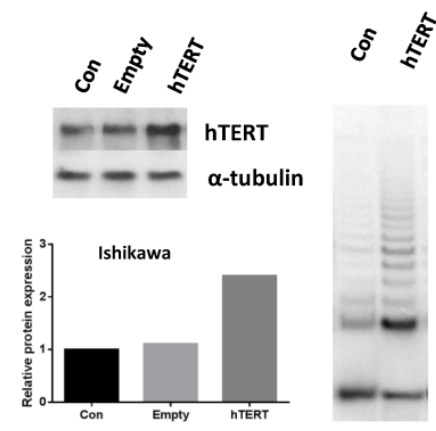

G

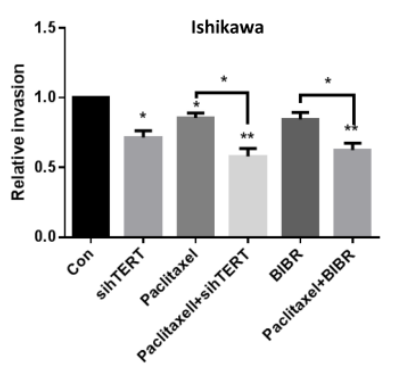

C
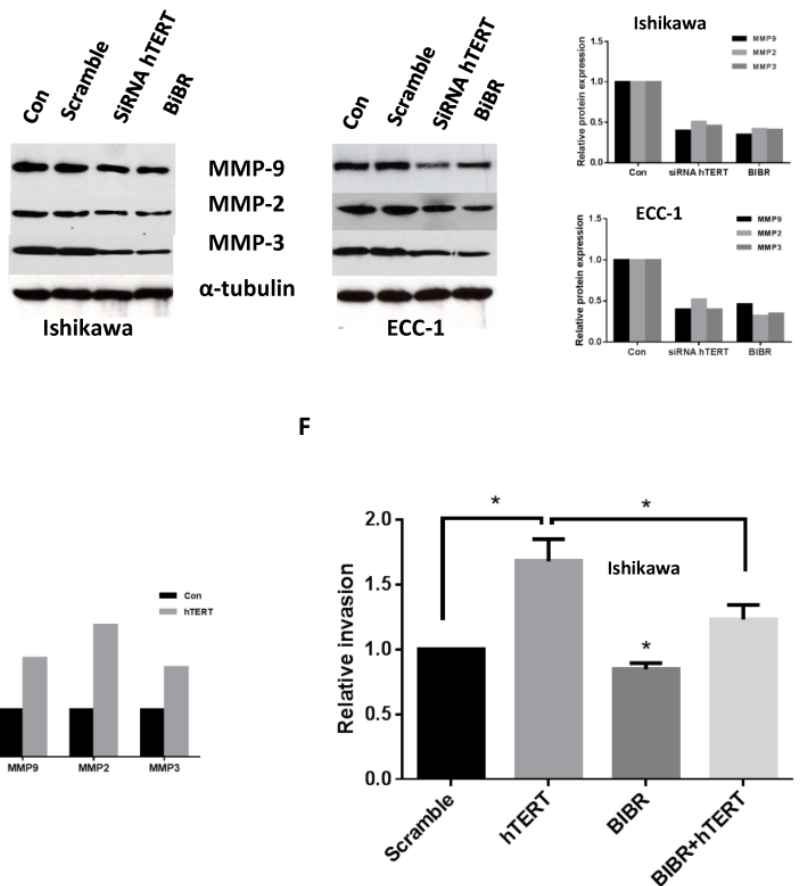

Figure 4. hTERT siRNA and BIBR 1532 inhibit cell invasion. ECC-1 and lshikawa cells were treated with hTERT siRNA and BIBR 1532 for 48 hours. Cell invasion was assessed by transwell assay (A and B). Western blotting showed hTERT siRNA and BIBR1532 decreased MMP2, MMP3 and MMP9 expression (C). Transfection Ishikawa cells with hTERT expression vector significantly increased telomerase activity (D), MMP protein expression (E) and cell invasion (F). Either hTERT siRNA or BIBR 1532 in combination with paclitaxel significantly decreased the ability of cell invasion in both cells $(\mathrm{G}$ and $\mathrm{H})$. Bar graphs show averages of 3 independent experiments $\pm \mathrm{SE}(* \mathrm{P}<0.05$, $* * \mathrm{P}<0.01)$.

\section{Discussion}

In the current study, we used two telomerase positive endometrial cancer cell lines, ECC-1 and Ishikawa, to address the role of knockdown hTERT by siRNA and inhibition of telomerase by BIBR1532 in cell growth and invasion. We showed that inhibition of telomerase activity by hTERT siRNA and BIBR1532 resulted in a decrease in cell growth alone with increased expression of cleaved caspase 3 and Annexin V. Knockdown of hTERT by siRNA and treatment of BIBR1532 decreased cell invasion in both cell lines, which led to downregulation of MMP2, MMP3 and MMP9 expression. The combination of hTERT siRNA or BIBR1532 with paclitaxel showed a synergistic effect in inhibition of cell proliferation, a significant increase in Annexin V positive cells and a remarkable decrease in cell invasion. In addition, overexpression of hTERT increased telomerase activity, enhanced the ability of invasion and blocked the inhibition of cell invasion induced by BIBR1532 in transfected Ishikawa cells. These results support the notion that hTERT regulates cell proliferation and invasiveness, and that targeting hTERT with molecular interventions may be a viable strategy for endometrial cancer therapy.

Accumulating evidence suggests that hTERT expression is specifically related to cancer cells and tightly coupled with telomerase activity. hTERT is the rate-limiting component for telomerase activation and expression of hTERT increases the proliferation rate and survival capacity of human cancer cells in vitro and in vivo [29-32]. Ectopic expression of hTERT promotes cell proliferation though multiple signaling pathways including EGFR, Wnt / $\beta$-catenin, c-Myc, NF-kB and PI3K/Akt signaling [31, 33]. Increased telomerase activity and hTERT expression in cancer cells have exhibited resistance to radiation and chemotherapeutic agents [34, 35]. Inhibition of hTERT expression by siRNAs or the small molecule inhibitor, 
BIBR1532, leads to a decrease in cell viability and an increase in chemosensitivity to doxorubicin, paclitaxel and adriamycin in tumor cells via induction of apoptosis $[8,29,36,37]$. Depletion of hTERT by siRNA sensitized cancer cells to chemotherapeutic agents which activated the mictochondrial apoptosis pathway [38]. These findings suggest that knockdown of hTERT was not only involved in the reduction of telomerase activity but also in augmentation of killing mechanisms of the chemotherapeutic agents. In agreement with previous studies on cell proliferation, inhibition of hTERT expression by siRNA or BIBR1532 led to an acute telomerase depletion and cell growth arrest, which was accompanied by increased annexin $\mathrm{V}$ and cleaved caspase 3 expression, ultimately leading to cell death in the endometrial cancer cells. hTERT siRNA or BIBR1532 mediated chemosensitizing effect was observed in paclitaxel treated cells. Thus, we believe that hTERT may be a potential target for a double-hit anticancer strategy [38].

The invasion and metastasis of cancer cells appear to be the consequence of a series of unique biological events within the tumor microenvironment, in part due to the degradation of basement membranes and extracellular matrix (ECM). The Matrix Metalloproteinases (MMP) family is responsible for degradation of the components of basement membranes and ECM $[39,40]$. MMP expression has been reported to be strongly related to advanced stage in endometrial cancer $[41,42]$. The levels of telomerase activity have a parallel association with migration and invasion in pancreatic cancer cells [43]. A recent study found that hTERT activates MMP9 transcription dependent on the NF-KB pathway, independently of status of telomerase activity in osteosarcoma U2OS cell line [44]. Overexpression of hTERT significantly promotes the invasive and metastatic potential of telomerase positive HepG2 cells via upregulation of mRNA and protein expression of MMP9 [39]. The knockdown of hTERT was correlated with reduction in expression of MMP9 and MMP2 and cell invasion in glioblastoma and neuroblastoma cells [34, 45]. In the present study, we noticed significant downregulation of MMP9, MMP2, and MMP3 expression, and reduction in invasion, after treatment with hTERT siRNA and BIBR1532. The combination treatment with paclitaxel led to a remarkable decrease in cell invasion and the expression of MMP proteins compared with monotherapy in both cell lines. Moreover, increased hTERT expression by transfection meaningfully enhanced the expression of MMP9, MMP2 and MMP3 and increased cell invasion. In addition, we also first confirmed that BIBR1532 effectively antagonized cell invasion induced by ectopic hTERT. These results sug- gest that hTERT mediated cell migration and invasion via the MMP family in endometrial cancer cells.

In conclusion, our study demonstrated that targeting hTERT by siRNA and BIBR1532 effectively inhibited cell proliferation and invasion of human endometrial cancer cells through induction of apoptosis and reduction of MMP protein expression. Inhibition of hTERT in combination with paclitaxel can produce a synergistic inhibitory effect in cell growth and a remarkable reduction in cell invasion. We believe our study establishes a causal relationship between inhibition of hTERT by siRNA and BIBR1532 and cell proliferation, apoptosis, and invasion in endometrial cancer cells, offering a potential therapeutic strategy for treatment of endometrial cancer.

\section{Competing interests}

No potential conflicts of interest were disclosed.

\section{References}

1. Ruden M, Puri N. Novel anticancer therapeutics targeting telomerase. Cancer treatment reviews. 2013; 39: 444-56.

2. Blackburn EH, Greider CW, Szostak JW. Telomeres and telomerase: the path from maize, Tetrahymena and yeast to human cancer and aging. Nature medicine. 2006; 12: 1133-8.

3. Zvereva MI, Shcherbakova DM, Dontsova OA. Telomerase: structure, functions, and activity regulation. Biochemistry Biokhimiia. 2010; 75: 1563-83.

4. Sprouse AA, Steding CE, Herbert BS. Pharmaceutical regulation of telomerase and its clinical potential. Journal of cellular and molecular medicine. 2012; 16: 1-7.

5. Lu MH, Liao ZL, Zhao XY, Fan YH, Lin XL, Fang DC, et al. hTERT-based therapy: a universal anticancer approach (Review). Oncology reports. 2012; 28: 1945-52.

6. Bashash D, Ghaffari SH, Zaker F, Hezave K, Kazerani M, Ghavamzadeh A, et al. Direct short-term cytotoxic effects of BIBR 1532 on acute promyelocytic leukemia cells through induction of p21 coupled with downregulation of c-Myc and hTERT transcription. Cancer investigation. 2012; 30: 57-64.

7. Marian CO, Cho SK, McEllin BM, Maher EA, Hatanpaa KJ, Madden CJ, et al. The telomerase antagonist, imetelstat, efficiently targets glioblastoma tumor-initiating cells leading to decreased proliferation and tumor growth. Clinical cancer research : an official journal of the American Association for Cancer Research. 2010; 16: 154-63.

8. Shi Y, Sun L, Chen G, Zheng D, Li L, Wei W. A combination of the telomerase inhibitor, BIBR1532, and paclitaxel synergistically inhibit cell proliferation in breast cancer cell lines. Targeted oncology. 2015.

9. Zhou C, Steplowski TA, Dickens HK, Malloy KM, Gehrig PA, Boggess JF, et al. Estrogen induction of telomerase activity through regulation of the mitogen-activated protein kinase (MAPK) dependent pathway in human endometrial cancer cells. PloS one. 2013; 8: e55730.

10. Bonatz G, Frahm SO, Klapper W, Helfenstein A, Heidorn K, Jonat W, et al. High telomerase activity is associated with cell cycle deregulation and rapid progression in endometrioid adenocarcinoma of the uterus. Human pathology. 2001; 32: 605-14.

11. Lehner R, Enomoto T, McGregor JA, Shroyer AL, Haugen BR, Pugazhenthi U, et al. Quantitative analysis of telomerase hTERT mRNA and telomerase activity in endometrioid adenocarcinoma and in normal endometrium. Gynecologic oncology. 2002; 84: 120-5.

12. Brustmann $\mathrm{H}$. Immunohistochemical detection of human telomerase reverse transcriptase (hTERT), topoisomerase IIalpha expression, and apoptosis in endometrial adenocarcinoma and atypical hyperplasia. International journal of gynecological pathology : official journal of the International Society of Gynecological Pathologists. 2005; 24: 184-92.

13. Boggess JF, Zhou C, Bae-Jump VL, Gehrig PA, Whang YE. Estrogen-receptor-dependent regulation of telomerase activity in human endometrial cancer cell lines. Gynecologic oncology. 2006; 103: 417-24.

14. Kurvinen K, Rantanen V, Syrjanen S, Johansson B. Radiation-induced effects on telomerase in gynecological cancer cell lines with different radiosensitivity and repair capacity. International journal of radiation biology. 2006; 82: 859-67.

15. Chen XJ, Zheng W, Chen LL, Chen ZB, Wang SQ. Telomerase antisense inhibition for the proliferation of endometrial cancer in vitro and in vivo. International journal of gynecological cancer : official journal of the International Gynecological Cancer Society. 2006; 16: 1987-93.

16. Meng E, Taylor B, Ray A, Shevde LA, Rocconi RP. Targeted inhibition of telomerase activity combined with chemotherapy demonstrates synergy in 
eliminating ovarian cancer spheroid-forming cells. Gynecologic oncology. 2012; 124: 598-605.

17. Parsch D, Brassat U, Brummendorf TH, Fellenberg J. Consequences of telomerase inhibition by BIBR1532 on proliferation and chemosensitivity of chondrosarcoma cell lines. Cancer investigation. 2008; 26: 590-6.

18. Mueller S, Hartmann U, Mayer F, Balabanov S, Hartmann JT, Brummendorf $\mathrm{TH}$, et al. Targeting telomerase activity by BIBR1532 as a therapeutic approach in germ cell tumors. Investigational new drugs. 2007; 25: 519-24.

19. Bashash D, Ghaffari SH, Zaker F, Kazerani M, Hezave K, Hassani S, et al. BIBR 1532 increases arsenic trioxide-mediated apoptosis in acute promyelocytic leukemia cells: therapeutic potential for APL. Anti-cancer agents in medicinal chemistry. 2013; 13: 1115-25.

20. Hanna RK, Zhou C, Malloy KM, Sun L, Zhong Y, Gehrig PA, et al. Metformin potentiates the effects of paclitaxel in endometrial cancer cells through inhibition of cell proliferation and modulation of the mTOR pathway. Gynecologic oncology. 2012; 125: 458-69.

21. Bae-Jump VL, Zhou C, Boggess JF, Whang YE, Barroilhet L, Gehrig PA Rapamycin inhibits cell proliferation in type I and type II endometrial carcinomas: a search for biomarkers of sensitivity to treatment. Gynecologic oncology. 2010; 119: 579-85.

22. Cantrell LA, Zhou C, Mendivil A, Malloy KM, Gehrig PA, Bae-Jump VL. Metformin is a potent inhibitor of endometrial cancer cell proliferation--implications for a novel treatment strategy. Gynecologic oncology. 2010; 116: 92-8

23. Shafer A, Zhou C, Gehrig PA, Boggess JF, Bae-Jump VL. Rapamycin potentiates the effects of paclitaxel in endometrial cancer cells through inhibition of cell proliferation and induction of apoptosis. International journal of cancer Journal international du cancer. 2010; 126: 1144-54.

24. Zhou C, Bae-Jump VL, Whang YE, Gehrig PA, Boggess JF. The PTEN tumor suppressor inhibits telomerase activity in endometrial cancer cells by decreasing hTERT mRNA levels. Gynecologic oncology. 2006; 101: 305-10.

25. Trentin L, Ballon G, Ometto L, Perin A, Basso U, Chieco-Bianchi L, et al. Telomerase activity in chronic lymphoproliferative disorders of B-cell lineage. British journal of haematology. 1999; 106: 662-8.

26. Zhou C, Gehrig PA, Whang YE, Boggess JF. Rapamycin inhibits telomerase activity by decreasing the hTERT mRNA level in endometrial cancer cells. Molecular cancer therapeutics. 2003; 2: 789-95.

27. Bieche I, Nogues C, Paradis V, Olivi M, Bedossa P, Lidereau $R$, et al. Quantitation of hTERT gene expression in sporadic breast tumors with a real-time reverse transcription-polymerase chain reaction assay. Clinical cancer research : an official journal of the American Association for Cancer Research. 2000; 6: 452-9.

28. Kondo N, Tsukuda M, Kimura M, Fujita K, Sakakibara A, Takahashi H, et al. Antitumor effects of telomelysin in combination with paclitaxel or cisplatin on head and neck squamous cell carcinoma. Oncology reports. 2010; 23: 355-63.

29. Dong X, Liu A, Zer C, Feng J, Zhen Z, Yang M, et al. siRNA inhibition of telomerase enhances the anti-cancer effect of doxorubicin in breast cancer cells. BMC cancer. 2009; 9: 133.

30. Kyo S, Takakura M, Fujiwara T, Inoue M. Understanding and exploiting hTERT promoter regulation for diagnosis and treatment of human cancers. Cancer science. 2008; 99: 1528-38.

31. Low KC, Tergaonkar V. Telomerase: central regulator of all of the hallmarks of cancer. Trends in biochemical sciences. 2013; 38: 426-34.

32. Smith LL, Coller HA, Roberts JM. Telomerase modulates expression of growth-controlling genes and enhances cell proliferation. Nature cell biology. 2003; 5: 474-9.

33. Wu XQ, Huang C, He X, Tian YY, Zhou DX, He Y, et al. Feedback regulation of telomerase reverse transcriptase: new insight into the evolving field of telomerase in cancer. Cellular signalling. 2013; 25: 2462-8.

34. George J, Banik NL, Ray SK. Knockdown of hTERT and concurrent treatment with interferon-gamma inhibited proliferation and invasion of human glioblastoma cell lines. The international journal of biochemistry \& cell biology. 2010; 42: 1164-73.

35. Xi L, Chen G, Zhou J, Xu G, Wang S, Wu P, et al. Inhibition of telomerase enhances apoptosis induced by sodium butyrate via mitochondrial pathway. Apoptosis : an international journal on programmed cell death. 2006; 11: 789-98.

36. Guo X, Wang W, Zhou F, Lu Z, Fang R, Jia F, et al. siRNA-mediated inhibition of hTERT enhances chemosensitivity of hepatocellular carcinoma. Cancer biology \& therapy. 2008; 7: 1555-60

37. Yu M, Kong H, Zhao Y, Sun X, Zheng Z, Yang C, et al. Enhancement of adriamycin cytotoxicity by sodium butyrate involves hTERT downmodulation-mediated apoptosis in human uterine cancer cells. Molecular carcinogenesis. 2014; 53: 505-13

38. Massard C, Zermati Y, Pauleau AL, Larochette N, Metivier D, Sabatier L, et al. hTERT: a novel endogenous inhibitor of the mitochondrial cell death pathway. Oncogene. 2006; 25: 4505-14.

39. Chen PC, Peng JR, Huang L, Li WX, Wang WZ, Cui ZQ, et al. Overexpression of human telomerase reverse transcriptase promotes the motility and invasiveness of HepG2 cells in vitro. Oncology reports. 2013; 30: 1157-64.

40. Sampieri CL, Leon-Cordoba K, Remes-Troche JM. Matrix metalloproteinases and their tissue inhibitors in gastric cancer as molecular markers. Journal of cancer research and therapeutics. 2013; 9: 356-63.

41. Weigel MT, Kramer J, Schem C, Wenners A, Alkatout I, Jonat W, et al. Differential expression of MMP-2, MMP-9 and PCNA in endometriosis and endometrial carcinoma. European journal of obstetrics, gynecology, and reproductive biology. 2012; 160: 74-8.

42. Planaguma J, Liljestrom M, Alameda F, Butzow R, Virtanen I, Reventos J, et al. Matrix metalloproteinase-2 and matrix metalloproteinase-9 codistribute with transcription factors RUNX1/AML1 and ETV5/ERM at the invasive front of endometrial and ovarian carcinoma. Human pathology. 2011; 42: 57-67.

43. Sato N, Mizumoto K, Nagai E, Tanaka M. Telomerase as a new target for pancreatic cancer treatment. Journal of hepato-biliary-pancreatic surgery. 2002; 9: 322-7

44. Ding D, Xi P, Zhou J, Wang M, Cong YS. Human telomerase reverse transcriptase regulates MMP expression independently of telomerase activity via NF-kappaB-dependent transcription. FASEB journal : official publication of the Federation of American Societies for Experimental Biology. 2013; 27: 4375-83.

45. Chakrabarti M, Banik NL, Ray SK. Sequential hTERT knockdown and apigenin treatment inhibited invasion and proliferation and induced apoptosis in human malignant neuroblastoma SK-N-DZ and SK-N-BE2 cells. Journal of molecular neuroscience : MN. 2013; 51: 187-98. 